\title{
Uber die Schreckreaktion bei Fischen und die Herkunft des Schreckstoffes
}

\author{
Von Wolfgang Pfeiffer
}

Zeitschrift für vergleichende Physiologie 43, 578-614 (1960)

Bei Hautverletzung einer Elritze (Phoximus laewis AG.) tritt aus der Wunde ein Schreckstoff aus. Er wird in starker Verdünnung von anderen Elritzen gerochen, veranlaßt sie zur Flucht und steigert ihre Wachsamkeit. Die geringe Menge Schreckstoff, die frei wird, wenn ein Hecht eine Elritze mit den Zähnen verletzt, ist ausreichend, um einen Schwarm zur Flucht zu veranlassen (K. v. Frisch, .1941). Eine Schreckstoffmenge, die einem $1 / 100 \mathrm{~mm}^{2}$ Hautfläche entspricht, genügt, um einen Elritzenschwarm in einem 14-Liter-Becken vom Futter zu vertreiben. Die Schreckreaktion ist angeboren. Sie ist bei Cyprinidae. Characidae und Cobitidae, auch bei nicht schwarmbildenden Arten, verbreitet. Barsche, Salmonidae und viele andere Süßwasserfische haben keine Schreckreaktion, trotz schwarmbildender Lebensweise. Die Wirksamkeit des Schreckstoffes einer Art auf eine andere Art ist zumindest bei den ennheimischen Cyprinidae eine allgemeine Erscheinung (F. Schuxz, 1956). Die Schreckreaktion hat bei Schwarmfischen die Bedeutung, den Schwarm vor größeren Verlusten durch räuberische Überfälle zu schützen (voN Frisch).

Während wir durch von FRISCH und SCHUrz über die Schreckreaktion bei Süßwasserfischen gut unterrichtet sind, fehlte eine Untersuchung der Meeresfische. Diese Lücke auszufüllen war das Ziel dieser Arbeit. Die genaue Kenntnis der Verbreitung der Schreckreaktion war auch Voraussetzung. um den Entstehungsort des Schredkstoffes in der Fischhaut zu finden.

Vor den Versuchen mit Meeresfischen wurden Versuche mit Süßwasserfischen gemacht. Dabei zeigte sich, daß3 auch Welse (Siluroidea) eine Schreckreaktion haben, die der Reaktion der Elritze gleichzusetzen ist, daß dagegen den Poecilidae, entgegen der Annahme von Schurz eine solche Reaktion fehlt. Die Sdiredkreaktion ist unter den Süßwasserfischen bei den Ostariophysen verbreitet.

An der Zoologischen Station Neapel, der Biologishen Station Arcachon (Gironde) und der Biologischen Anstalt Helgoland wurden von 1957 bis 1959 in mehr als 250 Versuchen an über 225 verschiedenen Versuchsgruppen und Schwärmen insgesamt 66 Arten Meeresfische aus 49 Gattungen und 32 verschiedenen Familien auf das Vorhandensein der Schreckreaktion hin untersucht. Das Ergebnis dieser Versuche war, besonders für ausgesprochene Schwarmfische, wie Clupeidae, Trachurus, Scomber, Belone, Ammodytes u, a., sehr überraschend: Allen geprüften Seefischen der europäischen Meere fehlt die Schreckreaktion. Das liegt daran, $\mathrm{da}$ alle diese Fischarten anderen Ordnungen als den Ostariophysen angehören. Der japanische Meerescyprinide Tribolodon (Syn.: Leuciscus) hakonensis taszanowskii (STEINDachner 1881) ist ein Beispiel dafür, dảß die Schreckreaktion auch in Seewasser möglich ist. Die Reaktionsschwelle dieses Fisches auf Schreckstoff gleicht der Schwelle der Elritzen. Elritzenhautextrakt wirkt schreckauslösend auf diesen Antipoden und ungekehrt. - Die Schreckreaktion ist bei den Ostariophysen verbreitet und auf diese Ordnung beschränkt. Nimmt man an, daß sie entstanden ist bevor sich die Ostariophysi in Siluroidea und Cyprinoidea gespalten haben, so ist ihre Entstehung etwa in Oligozän anzunehmen. Wenn die Schreckreaktion durch Selektionsdruck entstanden ist, mußten die "Ur-Ostariophysen" in Schwärmen leben, denn nur so konnte der Selektionsdruck ansetzen.

Einige Meeresfische haben eine optisch ausgelöste Fluchtreaktion: Sparidae bewahren eine Fluchtdistanz und sind durch Spreizen ihrer mit Hartstrahlen bewehrten Rückenflosse geschützt. Mugilidae fliehen bei Beunruhigung zur Wasseroberfäche und verharren dort ruhig. 
Um den Entstehungsort des Schreckstoffes in der Haut zu finden, wurden die Häute aller auf die Schreckreaktion hin untersuchten Fische histologisch miteinander verglichen. Für die Fixierung eigneten sich besonders die Gemische "Romeis" und "Bouin“, für die Färbung "Azan" und "Hämatoxylin-Eosin". Es zeigte sich, daß sich die Verbreitung der Schreckreaktion mit der Verbreitung einer bestimmten Art Kolbenzellen deckt. Die Literatur über Kolbenzellen der Fischhaut wurde revidiert: Verbreitung und Bedeutung dieser Zellen waren unbekannt. Die Kolbenzellen von Gadidae, Polypteridae, Carapus und Aal sind ihrer Funktion nach Schleimzellen. Nicht so die Kolbenzellen der Ostariophysi! Sie sind untereinander im Prinzip gleich. Die Kolbenzellen der Ostariophysi sind rund oder oval, nicht kolbenförmig. Sie haben keinen Ausführgang und werden nicht ausgestoßen. Um den zentralen Kern oder Doppelkern ist ein heller Hof. Daran schließt sich der färbbare Hauptinhalt. Die Kolbenzellen sind bei verschiedenen Arten unterschiedlich häufig. Sie können in einer oder mehreren Lagen vorkommen und sind gleichmäßig über den Körper verteilt. Nur auf den Fischbarteln sind sic sehr selten und klein.

Bei allen bisher untersuchten Arten mit Schreckstoff (29 Ostariophysen-Arten) konnte dieser Typ von Kolbenzellen nachgewiesen werden; bei keiner Fischart ohne Schredkstoff ( $82 \mathrm{Ar}$ ten ..Nicht-Ostariophysen") wurde er gefunden. Daß die Kolbenzellen nicht zur Epidermisoberfläche aufrücken und ihr Sekret nicht nach außen entleeren, stimmt damit überein, daß der Schreckstoff nur bei Hautverletzung frei wird. Ihre gleichmäßige Verteilung über den Fischkörper paßt zu dem Befund, daß die Haut äberall gleich wirksam ist. Die Barteln, die keine oder nur wenige kleine Kolbenzellen enthalten, liefern auch keinen oder nur sehr wenig Schreckstoff. All diese Befunde weisen darauf hin, daß die Kolbenzellen der Ostariophysi die Erzeuger des Schreckstoffes sind. Für sie wird deshalb der Name "Schredkstoffzellen" vorgeschlagen.

\section{Literatur}

1. Frisch, K. von: Ober einen Schreckstoff der Fischhaut und seine biologische Bedentung. $=Z$. vergl. Physiol. 29, 46-145 (1941).

2. Schutz, F.: Vergleichende Untersuchungen über die Schreckreaktion bei Fischen. $=\mathrm{Z}$. vergl. Physiol, 38, 84-135 (1956). 